\title{
Magnetic resonance imaging findings in patients with low backache
}

\author{
Narayan Bikram Thapa ${ }^{1}$, Suraj Bajracharya ${ }^{2}$ \\ ${ }^{1}$ Department of Radiology, KIST Medial College Teaching Hospital, Lalitpur, Nepal \\ ${ }^{2}$ Department of Orthopedics, KIST Medial College Teaching Hospital, Lalitpur, Nepal
}

Correspondence: Dr Narayan Bikram Thapa, KIST Medial College Teaching Hospital, Lalitpur, Nepal

Email: nadudoradio@yahoo.com

\begin{abstract}
Introduction: Low backache is commonly experienced by adults at some time during their lives. Though it is caused by degenerative changes, spinal stenosis, neoplasm, infection and trauma, lumbar disc degeneration is the most commonly diagnosed abnormalities associated. As Magnetic Resonance Imaging (MRI) is non invasive imaging technique with excellent spatial and contrast resolution, it has become the investigation of choice in evaluation of patients with low back pain. This study was designed to determine the patterns of degenerative disc disease on MRI in patients with low backache.

Methods: A retrospective hospital based study was done by reviewing MRI report of 202 patients who underwent MRI of lumbar spine for complaint of chronic low back pain, radicular pain, neurogenic claudication or various other symptoms and signs suggestive of lumbar degenerative disc disease from January 2014 till June 2014. The patients having MRI findings of acute spinal infection, recent trauma, tumors, spinal dysraphism and metabolic conditions were excluded from the study.

Results: Out of the 202 patients included in the study, 116 patients (57.4\%) were male and 86 patients $(42.6 \%)$ were female. The mean age of the study population was $44.26 \pm 15.61$ (13-83) years. Multiple contiguous level disc disease was the most common type of involvement which was noted in 109 (54\%) patients. Grade 4 lumbar disc degeneration (graded as per classification given by Pfirrmann et al) was noted in $65.3 \%$ (132) cases followed by Grade 2 in $25.2 \%(51)$ cases and Grade 1 in $5.5 \%$ (11) cases. The most common involvement was observed at L4-L5 level (76.7\%) and L5-S1 levels (55.9\%) followed by L3-L4 (30.6\%) in decreasing order of frequency. The most common category was disc bulge note in $46.5 \%$ (94) of cases. Nerve root compression was observed in $56 \%$ (114) of the total cases. Nerve root compromise was also noted most frequently compressing L5 nerve (28.23\% of cases). Annular tear was observed in $14.4 \%$ (29) of cases and among them $20 \%$ (6) of cases had torn at two intervertebral discs. It is most frequently noted involving L4-L5 intervertebral disc ( $72.5 \%$ of cases), followed by L5-S1 ( $24.2 \%$ of cases).
\end{abstract}

Conclusion: Disc generation is most common at L4-L5 level with multiple contiguous involvement of grade 4. Annular tear though not common can occur and is also common at L4-L5 level.

Keywords: Annular tear; disc degeneration; low back ache; modic changes 


\section{Introduction}

Low back ache is one of the common indications for MRI in our clinical practice. It is estimated that $70-80 \%$ of adults experience low backache at some time during their lives. ${ }^{1}$ The causes of low back pain include degenerative changes, spinal stenosis, neoplasm, infection, trauma, and inflammatory or arthritic processes. Among these, lumbar disc degeneration is the most commonly diagnosed abnormalities associated with low back pain. ${ }^{2}$ There are varieties of factors that contribute to this condition. Aging, axial loading of disc, vascular in growth, and abnormalities in collagen and proteoglycan all contribute to disc degeneration. Disc herniation with radiculopathy and chronic discogenic low back pain are the result of this degenerative process. ${ }^{3}$

MRI is non invasive imaging technique with excellent spatial and contrast resolution. Hence, it has become the investigation of choice in evaluation of patients with low back pain or radicular pain. It has also emerged as an investigation of choice over the other investigations for a herniated disc and become a gold standard to diagnose herniated disc. ${ }^{4}$ MRI is also useful in planning surgical management of patients with sciatica attributable to lumbar disc herniation. ${ }^{5}$ Hence, MRI is the emerging radiological diagnostic tool in the management of low back pain. However, MRI facility is not available in all the part of the country and even in the places where this investigation is available, being one of the expensive investigations, it might be beyond affordability for most of the Nepalese population. Till date, not many studies have been done in our geographical region to identify spectrum of lumbar disc pathology prevalent in this part of the world. Hence, this study was designed to determine retrospectively the patterns degenerative disc disease on MRI in patients with low back pain. The result of the study might have clinical relevance about what is to be expected in the patient with low back ache due to degenerative changes.

\section{Material}

This is hospital based retrospective study of 202 MRI reports of lumbar spine, done at Capital Hospital, Kathmandu, Nepal. Patients who underwent MRI lumbar spine for complaints of chronic low back pain, radicular pain, neurogenic claudication or various other symptoms and signs suggestive of lumbar degenerative disc disease from January 2014 till June 2014 were included in the study. Those patients having MRI findings of acute spinal infection, recent trauma, tumors, spinal dysraphism and metabolic conditions were excluded from the study.
The MRI examination was done on 0.3T Hitachi, AirisVentos with acquisition of the T1W, T2W, STIR images in sagittal, axial and coronal planes. The following criteria were evaluated on MRI: lumbar disk degeneration (graded as per classification given by Pfirrmann et al), disc herniation (graded as normal, bulge, protrusion, extrusion and sequestration), presence of annular tear, nerve root compromise (graded as per Pfirrmann et al classification), degenerative vertebral endplates changes (graded as per Modic classification). ${ }^{6,7,8}$ All the MRI reporting was done by the principal investigator. Permission for the retrospective review was taken from the hospital management.

\section{Result}

Out of the 202 patients included in the study, 116 patients $(57.4 \%)$ were male and 86 patients $(42.6 \%)$ were female. The mean age of the study population was $44.26 \pm 15.61$ (1383 ) years, $19.3 \%$ (39) were less than 39 years, $38.1 \%$ (77) were in 40-59 years age group and $42.6 \%(86)$ older than 60 years.

Multiple contiguous level disc disease was noted in 109 (54\%) patients, multiple level disc disease with skipped segments was noted in $19(9.4 \%)$ patients, while 74 $(36.6 \%)$ patients in the study group had single level disc involvement. Grade 4 lumbar disc degeneration (graded as per classification given by Pfirrmann et al)was noted in $65.3 \%$ (132) cases followed by Grade 2 in $25.2 \%(51)$ cases and Grade 1 in 5.5\% (11) cases.

The incidence of various categories of disc herniation at different levels was calculated and presented in the figure 1. The most common involvement was observed at L4-L5 level $(76.7 \%)$ and L5-S1levels (55.9\%) followed by L3L4 (30.6\%) in decreasing order of frequency. The most common category was disc bulge note in $46.5 \%$ (94) of cases involving L4-L5 disc.

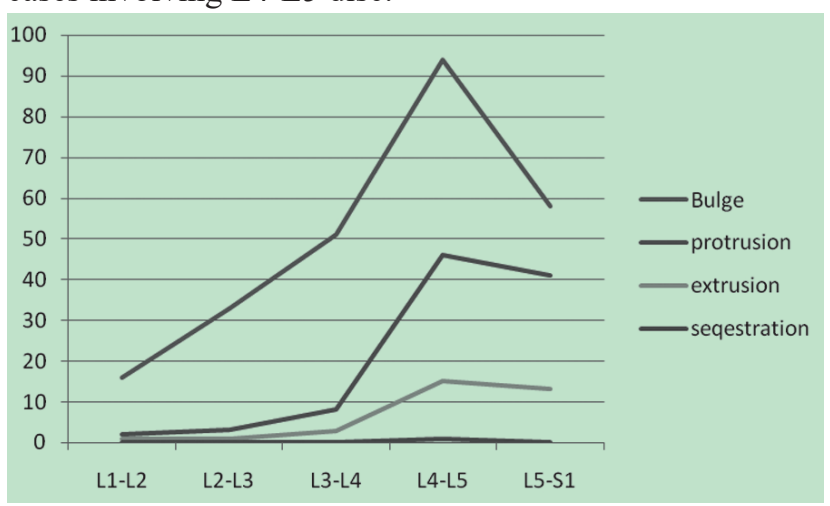

Figure 1. Various categories of disc herniation at different levels 
Table 1. Grades of nerve root compression at different level

$\begin{array}{llll}\text { Compressed nerve } & \text { Grade } 1 & \text { Grade 2 } & \text { Grade 3 } \\ \text { L2 } & & & 3 \\ \text { L3 } & 2 & 3 & 4 \\ \text { L4 } & 2 & 5 & 10 \\ \text { L5 } & 2 & 17 & 38 \\ \text { S1 } & 5 & 15 & 31\end{array}$

Nerve root compression was observed in 55.9\% (113) of the total cases. Multiple nerve compression was observed in $7.07 \%$ (8) cases. Among them, central canal stenosis was observed in $70 \%$ (79), neural foraminal narrowing in $35.4 \%$ (40). Nerve root compromise was also noted most frequently at L4-L5 level (28.23\% of cases),followed by L5-S1 (25.25\%) and L3-L4 (8.4\%) levels in decreasing order of frequency as presented in the table 1 which shows various grades of nerve root compromise at respective disc levels. Grade 3 nerve root compression was the most common and was observed in $76 \%$ (86) of cases with nerve root compression. Figure 2, 3, 4 demonstrates common MRI findings observed in this study.

Annular tear was observed in $14.4 \%$ (29) of cases and among them, 20\% (6) of cases had tear at two intervertebral discs. It is most frequently noted involving L4-L5 intervertebral disc $(72.5 \%$ of cases), followed by L5-S1 (24.2\% of cases), L3-L4 (17.3\% of cases) and L2-L3 (0.5\% of cases) respectively.

Vertebral end plate changes were seen only in $7.4 \%$ of cases (15) and most commonly at L5-S1 and L4-L5 vertebral levels with Modic Type II changes (80\% of cases) being most common followed by Type I changes (46.6\%). Type III changes were not identified in any of the patients in the study group.

Figure 2

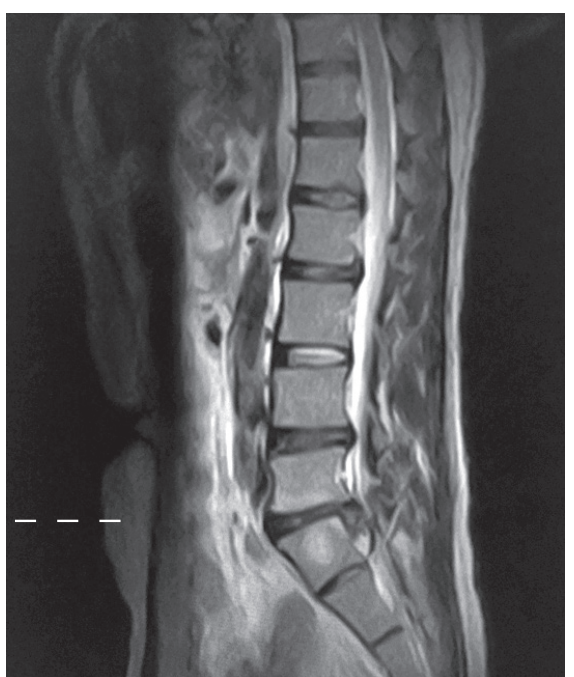

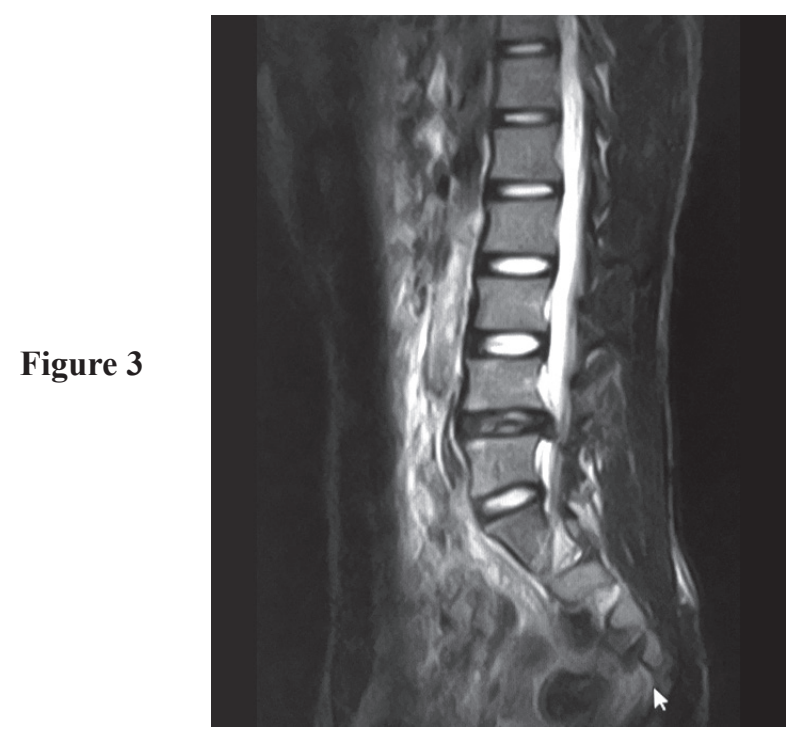

Figure 4

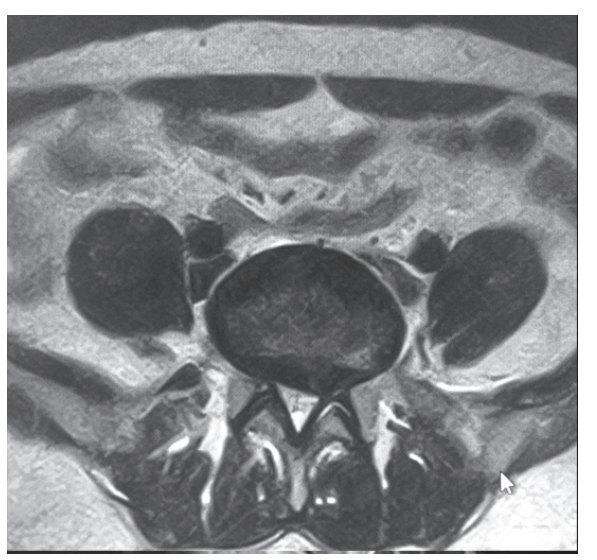

Figure 2: T2W Sagital image with disc desiccations at L4-L5 and L5-S1 level. Diffuse bulge noted at L4-L5 level and posterior-central extrusion noted at L5-S1 level (White arrow) causing severe narrowing of the central spinal canal.

Figure 3: T2W Sagital image shows disc desiccation at L4-L5 level with posterior-central protrusion causing narrowing of the central spinal canal

Figure 4: T2W axial image shows left paracentral protrusion causing narrowing of the central spinal canal and left lateral recess. The disc is impinging left traversing nerve root.

\section{Discussion}

This retrospective study demonstrated the spectrum of MRI findings in patients with low back pain in our setting. The mean age for presentation with low back pain was 44.26 years. The age of patient presenting with low backache is 
in accordance with other studies (Horal, Ansari MA, and Suk et al). ${ }^{9-11}$ In this study, $57.4 \%$ of patients were male. The male predominance was also found in the observations made by Horal $^{9}$ and Ansari MA et al ${ }^{10}$.

In this study, the most common involvement was observed at L4-L5 level (76.7\%) and L5-S1levels (55.9\%). This is consistent with most abnormal MRI findings at the lowest lumbar levels (L4-L5 \& L5-S1). In the study done by S Verma et al, ${ }^{12}$ in patient between $20-40$ years, the incidence of disc degeneration was most frequent at L4-L5 level (79.3\% of cases) followed by L5-S1 level (68.9\%).

Multiple contiguous level disc disease was the most common involvement, noted in 109 (54\%) patients followed by single level disc involvement which was noted in $74(36.6 \%)$ patients in the present study. This finding is similar to the finding of the study done by S Verma ${ }^{12}$ who observed multiple contiguous level disc disease in 138 $(59.5 \%)$ patients and single level disc involvement in 52 $(22.4 \%)$ patients.

In this study, nerve root compression was observed in $55.9 \%$ (113) of the total cases. Among them, central canal stenosis was observed in 70\% (79), neural foraminal narrowing in $35.4 \%$ (40). Yong et al ${ }^{13}$ conducted study in 56 Japanese patients where they observed spinal stenosis in $34(59.6 \%)$ and foramina narrowing in $17(29.8 \%)$ patients. Higher incidence of compressive lesions in theirs' study compared to the present one may be due to racial difference. In the similar study done by Ansari MA et al ${ }^{10}$ in 301 Nepalese patients, nerve root compression was seen in only $37.8 \%$ of the population, neural foraminal stenosis was the commonest complication of degenerative changes seen in $68.4 \%$ of the population and central spinal canal stenosis was seen in $51.8 \%$ of population. Higher incidence of nerve root compression and different type of stenosis observed in present study could be due to enrollment of older patients in the present study. However, wheather central canal stenosis or foraminal stenosis is common in Nepalese population need to be confirmed by further study with larger sample size.

Findings of disc degeneration are often interpreted as causes of back pain, triggering both medical and surgical interventions, which are sometimes unsuccessful in alleviating the patient's symptoms. ${ }^{14}$ Prior studies have demonstrated that imaging findings of spinal degeneration associated with back pain are also present in a large proportion of asymptomatic individuals ${ }^{15,16}$. Hence, further study comparing MRI findings in the symptomatic and asymptomatic patients would enlighten the clinical significance of degenerative changes in MRI. The Limitations of the study were that it was a retrospective study, there were less number of patients and there would be variation in reporting by different radiologist. Conclusion: This study shows spectrum of MRI findings in patients with low back ache. Disc generation is most common at L4-L5 level with multiple contiguous involvement of grade 4. Annular tear though not common can occur and is also common at L4-L5 level.

\section{References}

1. Olmarker K, Rydevik B. Pathophysiology of sciatica. Orthop Clin North Am. 1991;22:223-34.

2. Frymoyer JW, Pope MH, Clements JH, Wilder DG, MacPherson B, Ashikaga T. Risk factors in low-back pain. An epidemiological survey. J Bone Joint Surg Am. 1983;65:213-8.

3. Martin MD, Boxell CM, Malone DG. Pathophysiology of lumbar disc degeneration: a review of the literature. Neurosurg Focus 2002;13(2):E1.

4. Cranshaw C, Kean DM. The use of unclear MR in diagnoses of lateral canal extra percent. J Bone Joint Surg Am. 1984;66B:711-4.

5. Carragee, E.J. and D.H.A. Kim, 1997. Prospective Analysis of Magnetic Resonance Imaging Findings in Patients with Sciatica and Lumbar Disc Herniation. Correlation of Outcomes with Disc Fragment and Canal Morphology. Spine (Phila Pa 1976),22(14): 1650-1660.

6. Pfirrmann CW, Dora C, Schmid MR, et al. MR imagebased grading of lumbar nerve root compromise due to disk herniation: reliability study with surgical correlation. Radiology 2004;230:583-8.

7. Modic MT, Steinberg PM, Ross JS, et al. Degenerative disk disease: assessment of changes in vertebral body marrow with MR imaging. Radiology 1988;166:1939.

8. Horal J. The clinical appearance of low back disorders in the city of Gothenburg, Sweden. Comparison of incapacitated probands with matched controls. Acta Orthop Scand Suppl. 1969;118:1-109.

9. Ansari MA, Subedi K, Panta OB, Suwal S. MRI Pattern of Lumbosacral Degeneration in Tribhuvan University Teaching Hospital, Nepal. Journal of Institute of Medicine, August, 2015, 37:2: 51-55 
10. Suk KS, Lee HM, Moon SH, Kim NH. Lumbosacral scoliotic list by lumbar disc herniation. Spine (Phila Pa 1976) 2001;26:667-71.

11. S Verma, P Gupta, A Munshi, P Goyal, S Verma, V Sardana. A Retrospective Analysis Of Magnetic Resonance Imaging Findings In 20 -40 Year Old Patients With Low Back Pain. Experience At A Semi Urban Tertiary Healthcare Centre In Northern India.. The Internet Journal of Spine Surgery. 2010 Volume 6 Number 1.

12. Yong, P.Y., N.A.A. Alias and I.L. Shuaib, 2003. Correlation of Clinical Presentation, Radiography and Magnetic Resonance Imaging for Low Back Pain- A Preliminary Survey Resonance Imaging for Low Back Pain. Journal of Hong Kong College of Radiology, 6(23): 144-151.

13. Carragee E, Alamin T, Cheng I, et al. Are first-time episodes of serious LBP associated with new MRI findings? Spine J. 2006;6:624-35.

14. Boden SD, Davis DO, Dina TS, et al. Abnormal magnetic-resonance scans of the lumbar spine in asymptomatic subjects: a prospective investigation. J Bone Joint Surg Am. 1990;72:403-08.

15. Kalichman L, Kim DH, Li L, et al. Computed tomography-evaluated features of spinal degeneration: prevalence, intercorrelation, and association with selfreported low back pain. Spine J. 2010;10:200-08.

16. Wiesel SW, Tsourmas N, Feffer HL, et al. A study of computer-assisted tomography. I. The incidence of positive CAT scans in an asymptomatic group of patients. Spine (Phila Pa 1976) 1984;9:549-51. 